\title{
Influence of crystal anisotropy on elastic deformation and onset of plasticity in nanoindentation - a simulational study
}

\author{
Gerolf Ziegenhain, ${ }^{1}$ Herbert M. Urbassek, ${ }^{1, *}$ and Alexander Hartmaier ${ }^{2}$ \\ ${ }^{1}$ Fachbereich Physik und Forschungszentrum OPTIMAS, Universität Kaiserslautern, \\ Erwin-Schrödinger-Straße, D-67663 Kaiserslautern, Germany \\ ${ }^{2}$ Interdisciplinary Center for Advanced Materials Simulation (ICAMS), Ruhr-University Bochum, \\ Stiepeler Str. 129 (UHW), D-44780 Bochum, Germany
}

(Dated: January 15, 2014)

\begin{abstract}
Using molecular-dynamics simulation we simulate nanoindentation into the three principal surfaces - the (100), (110) and (111) surface - of $\mathrm{Cu}$ and $\mathrm{Al}$. In the elastic regime, the simulation data agree fairly well with the linear elastic theory of indentation into an elastically anisotropic substrate. With increasing indentation, the effect of pressure hardening becomes visible. When the critical stress for dislocation nucleation is reached, even the elastically isotropic Al shows a strong dependence of the surface orientation on the force-displacement curves. After the load drop, when plasticity has set in, the influence of the surface orientation is lost, and the contact pressure (hardness) becomes independent of the surface orientation.
\end{abstract}

PACS numbers: $62.20 .-\mathrm{x}, 81.40 . \mathrm{Jj}$

Keywords: Molecular dynamics, hardness, nanoindentation, interatomic potentials, plasticity, elasticity

\section{INTRODUCTION}

In a seminal paper, Hertz investigated the elastic interaction between a sphere of radius $R$ with an elastic isotropic solid. ${ }^{1}$ He found that the force $F$ acting on the sphere normally to the surface and the displacement $d$ into the surface are related by the so-called Hertz law

$$
F=\frac{4}{3} M d^{3 / 2} \sqrt{R}
$$

In this relation, a single materials parameter, the so-called indentation modulus $M$ describes the materials elastic response. ${ }^{1-4}$ For a rigid indenter, it may be expressed in terms of the Young's modulus $E$ and the Poisson ratio $\nu$ of the substrate as

$$
M=\frac{E}{1-\nu^{2}} .
$$

Hertz also determined the contact pressure; it is defined by the ratio of the normal force $F$ divided by the contact area projected into the surface plane. Hertz obtained

$$
p=\frac{4}{3 \pi} M \sqrt{\frac{d}{R}} .
$$

We note that Hertz' analysis is exact in the limit of $d \ll R$.

Nowadays indentation experiments can be performed into single crystals. ${ }^{5,6}$ The question then arises in how far Hertz' analysis also describes the indentation of crystalline - and thus by definition anisotropic - materials. An analytical extension of Hertz' analysis to anisotropic materials is, however, non-trivial. Willis ${ }^{7,8}$ appears to have gone farthest in the analysis; however, no analytical results are available, and one has to resort to numerical procedures even in the simplest case, i.e., for transversally isotropic media. Vlassak and $\mathrm{Nix}^{9,10}$ evaluated these results numerically for the specific problems of a flat circular punch and an axisymmetric paraboloid indenter; the latter approximates a spherical indenter for small indentation depths. They showed that in this case the Hertzian law Eq. (1) holds with a modified indentation modulus $M(\boldsymbol{\Omega})$ which depends on the surface orientation $\boldsymbol{\Omega}$, and calculated this quantity numerically. In their experiments on Au single crystals, Kiely and Houston found considerable deviations between the experimental results on a Au substrate and the theoretical predictions. ${ }^{5}$ Recently, Tsuru and Shibutani employed molecular-dynamics (MD) simulations to study indentation into an anisotropic half-space, and demonstrated that indentation into fcc crystals indeed depends on the surface orientation. ${ }^{11,12}$ Other authors also investigated the 
question in how far the homogeneous nucleation of dislocations under the indenter, i.e., the onset of plasticity, is influenced by the surface orientation. ${ }^{13-15}$

In the present paper, we study these questions for two fcc materials, $\mathrm{Al}$ and $\mathrm{Cu}$, which show a widely differing anisotropy. This allows us to discuss quantitatively in how far the Hertzian law, (1), is obeyed. Finally, we demonstrate that with increasing penetration into the target, the indenter measures the bulk hardness of the system and the surface orientation loses its influence.

\section{METHOD}

\section{A. Simulation}

We chose two different fcc materials, $\mathrm{Al}$ and $\mathrm{Cu}$, for our study, which differ strongly in their elastic anisotropy. Many-body potentials of the embedded-atom type are used to model these metals; both the $\mathrm{Al}$ potential ${ }^{16}$ and the $\mathrm{Cu}$ potential $^{17}$ reproduce the zero-temperature elastic moduli given in Table I. The fcc substrate has approximately cubic shape with side lengths of around $25 \mathrm{~nm}$; it contains roughly $1.35 \times 10^{6}$ atoms. We checked in a series of simulations that our crystallite size is large enough to obtain reliable results for the indentation process. We found that a careful relaxation of the crystal before starting the indentation process to $p_{i j}<10^{-5} \mathrm{GPa}$ and temperatures $\ll 1 \mathrm{~K}$ was mandatory to obtain reliable and reproducible results. Lateral periodic boundary conditions have been applied. At the bottom, atoms in a layer of the width $r_{\text {cut }}$ have been constrained to $F_{\text {normal }}=0$.

The indenter is modelled as a repulsive sphere. We chose a non-atomistic representation of the indenter. Its interaction potential with the substrate atoms is described by ${ }^{18}$

$$
V(r)= \begin{cases}k(R-r)^{3}, & r<R, \\ 0, & r \geq R .\end{cases}
$$

The indenter radius was set to $R=8 \mathrm{~nm}$, and the indenter stiffness to $k=3 \mathrm{eV} / \AA^{3}$. We checked that our results are only weakly influenced by the exact value of the contact stiffness, as long as it is in the range of $1-10 \mathrm{eV} / \AA^{3}$.

The simulations have been performed using a modified version of the LAMMPS code, ${ }^{19}$ using the so-called displacement-controlled approach. ${ }^{20,21}$ The indenter is advanced every $\Delta t=2$ ps by a fixed amount of $\delta=0.256$ $\AA$ (《 lattice constant) instantaneously, corresponding to an average indentation speed of $v=12.8 \mathrm{~m} / \mathrm{s}$. The substrate then relaxes for the ensuing time of $\Delta t$ to the new indenter position.

\section{B. Elastic properties}

The materials considered here crystallize in the cubic fcc structure. Their elastic behaviour is therefore completely described by their elastic constants, $c_{11}, c_{12}$, and $c_{44}$. Their zero-temperature values, are given in Table I. These two materials differ in particular in their elastic anisotropy, defined as

$$
X=\frac{2 c_{44}}{c_{11}-c_{12}}
$$

which measures both the orientation dependence of the elastic modulus and of the shear moduli. As Table I shows, $\mathrm{Cu}$ is quite anisotropic, $X=3.22$, while $\mathrm{Al}$ is nearly isotropic, $X=1.20$. Fig. 1 demonstrates the anisotropy of the two materials by plotting Young's modulus, $E(\boldsymbol{\Omega})$, in its dependence on orientation $\boldsymbol{\Omega}$. It is given by

$$
\frac{1}{E(\boldsymbol{\Omega})}=\frac{1}{E}-\frac{1}{c_{44}}(X-1) \Gamma(\boldsymbol{\Omega}),
$$

where $E$ is the orientation-averaged Young's modulus, which is related to the average shear modulus $G$ (cf. Eq. (10)) and the average Poisson ratio $\nu$ (cf. Eq. (11)) via

$$
E=2 G(\nu+1)
$$

and 


$$
\Gamma(\boldsymbol{\Omega})=\Omega_{1}{ }^{2} \Omega_{2}{ }^{2}+\Omega_{2}{ }^{2} \Omega_{3}{ }^{2}+\Omega_{3}{ }^{2} \Omega_{1}{ }^{2}
$$

describes the angular dependence. ${ }^{22}$

For reference purposes, it is interesting to define pertinent isotropic properties, such as they would apply to a polycrystalline sample: ${ }^{9}$ the bulk modulus $B$,

$$
B=\frac{c_{11}+2 c_{12}}{3},
$$

an average shear modulus $G$, defined as the arithmetic mean over the Voigt- and Reuss-averaged shear moduli

$$
G=\frac{1}{2}\left(\frac{c_{11}-c_{12}+3 c_{44}}{2(G+3 B)}+\frac{5\left(c_{11}-c_{12}\right) c_{44}}{4 c_{44}+3\left(c_{11}-c_{12}\right)}\right),
$$

and an average Poisson ratio $\nu$ as appropriate for an isotropic solid

$$
\nu=\frac{3 B-2 G}{2(G+3 B)}
$$

Indentation moduli $M(\boldsymbol{\Omega})$ for the three crystal orientations have been determined numerically by Vlassak and Nix. ${ }^{9,10}$ We evaluate these moduli for $\mathrm{Al}$ and $\mathrm{Cu}$ and give the results, denoted as $M^{\text {theo }}$ in Table II. The orientation dependence amounts to only $1 \%$ for $\mathrm{Al}$, while for $\mathrm{Cu}$, deviations of up to $10 \%$ from the isotropic value, $M^{\text {iso }}$, show up. In agreement with the orientation dependence of the Young's modulus, Fig. 1, the (111) plane is the stiffest, and the (100) plane the softest; the (110) plane is intermediate, and its modulus is quite close to the isotropic value.

Other authors ${ }^{12}$ attempt a different procedure and define

$$
M^{\operatorname{appr}}(\boldsymbol{\Omega})=\frac{E(\boldsymbol{\Omega})}{1-\nu^{2}(\boldsymbol{\Omega})} .
$$

Thus they use the definition of the isotropic indentation modulus Eq. (2), and replace Young's modulus $E$ by the orientation dependent Young modulus $E(\boldsymbol{\Omega})$. Analogously, $\nu$ is replaced by the Poisson ratio $\nu(\boldsymbol{\Omega})$, which characterizes the contraction transverse to $\boldsymbol{\Omega}$; for the (100) and (111) orientation, this value is uniquely defined, while for the (110) direction a Voigt average is applied. The resulting values of this approximated indentation modulus are displayed in Table II. They show a considerably larger anisotropy than the Vlassak-Nix moduli; the deviation from the isotropic modulus vary between -9 and $+6 \%$ for $\mathrm{Al}$ and between -44 and $+45 \%$ for Cu. Evidently, $M^{\text {appr }}$ is not a good approximation to the true theoretical value, $M^{\text {theo }}$.

\section{RESULTS}

\section{A. Elastic regime}

In Fig. 2 we show the force-displacement curves for $\mathrm{Al}$ and $\mathrm{Cu}$ indented on the three principal surface planes. In the elastic regime, which is characterized by the $d^{3 / 2}$ dependence of the force and terminates with a more or less drastic force maximum at a depth of around $6-8 \AA$, $\mathrm{Cu}$ is stiffer than $\mathrm{Al}$, corresponding to the larger elastic moduli of $\mathrm{Cu}$. Both materials show a dependence on the surface orientation, such that the stiffness is highest for the (111) plane and smallest for the (100) plane, in agreement with the orientation dependence of the indentation modulus, cf. Table II. The orientation dependence is most pronounced for $\mathrm{Cu}$, corresponding to the larger anisotropy $X$ of this material.

We fit the elastic part of the force-indentation curve to the Hertzian law, Eq. (1), using $M$ as a fit parameter. We

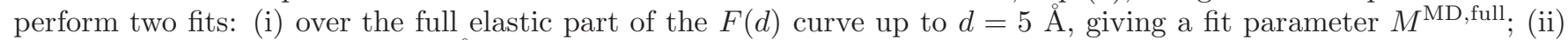
a fit only the initial part, $d<2 \AA$, giving $M^{\mathrm{MD} \text {,init }}$. The fit values are included in table II. We see that the full fit gives consistently larger fitted indentation moduli than the initial fit, $M^{\mathrm{MD} \text {,full }}>M^{\mathrm{MD} \text {,init }}$. This is a sign of the onset of nonlinear elasticity: under pressure, a material shows an increased stiffness; this feature has been termed pressure hardening. ${ }^{23,24}$ This effect of pressure hardening has been demonstrated previously by comparing FEM simulations of the indentation process using linear vs nonlinear elasticity. ${ }^{23}$ The fact that the indenter is not exactly a hard sphere, 
but - for numerical reasons - has to be defined with a finite stiffness in form of the polynomial indenter potential, Eq. (4), also causes the initial stiffness to be systematically smaller than the stiffness at larger penetration depths; this feature enhances the effect of the pressure hardening.

A comparison of the simulation results with theory hence must be based on the initial fit values $M^{\mathrm{MD} \text {,init }}$. Table II demonstrates a fairly good agreement between theory and simulation; the largest deviations amount to $14 \%$. We note that - with the exception of the $\mathrm{Cu}(100)$ surface - all fit values are above the theoretical values; this points at the possibility that even for the small fit regime of $0<d<2 \AA$, already some departures from linear elasticity may show up. Among other reasons which may contribute to the deviations we mention the atomistic nature of the indentation process, which for the indenter radius of $8 \mathrm{~nm}$ is not fully captured by continuum elasticity, and also the numerical problem of fitting the molecular-dynamics data to the Hertzian law - note that besides the elastic deformation also a finite offset in the displacement has to be fitted.

In Fig. 3 we give a graphical representation of the comparison between MD simulation results, the fit curves, and the theoretical prediction for the case of the (100) surfaces. Both for $\mathrm{Al}$ and $\mathrm{Cu}$, the $\mathrm{MD}$ data are almost indistinguishable from the full fit. It is also evident that deviations between the full fit and the initial fit become sizable only for $d>2$ $\AA$, as it must be. The approximate indentation moduli, Eq. (12), severely underestimate the simulation data, while the theoretical prediction by Vlassak and Nix gives a fair representation of the simulation data.

\section{B. Plasticity}

The onset of plasticity is visible in the force-displacement curves of Fig. 2 by the pronounced load drop appearing for the (111) and - to a lesser extent - for the (110) surface. In the following we denote the stress at which this load drop appears as the critical stress and the pertinent indentation as the critical indentation depth. For the (100) surface, dislocation nucleation sets in considerably earlier and in a more continuous way, such that any load drop is blurred out.

These principal differences between the (100) and (111) surfaces and the abrupt onset of plasticity for the (111) surface can be explained as follows: (i) the primary glide systems $\langle 1 \overline{1} 0\rangle\{111\}$ are located at quite oblique angles to the direction of the indentation force acting normally to the surface; the corresponding Schmid factor is only $s_{111}=\sqrt{2 / 27}=0.27$. For the (100) surface these glide systems are more easily activated, since $s_{100}=1.5 s_{111}=$ $1 / \sqrt{6}=0.41$. (ii) When finally the critical indentation depth has been reached, a considerable elastic energy has built up due to the high stiffness of this surface. Then, upon dislocation nucleation, a stronger dislocation avalanche and consequently a larger plastic displacement jump are achieved. The (110) surface shows a somewhat more complex indentation behaviour; while the pertinent Schmid factor is identical to the (100) surface, the onset of plasticity rather follows the (111) surface. We assume that this is due to the complex stress distribution found below this surface; in particular, the maximum shear stress is not below the indenter along the indentation axis but rather shifted to the side; we also observe more than one primary nucleation site for dislocations. Note also that it has been shown ${ }^{14,25}$ that - in single-crystalline $\mathrm{Cu}$ - non-Schmid factors may be important in describing dislocation nucleation for uniaxial tension in [110] direction, but not in [111] or in [100] direction.

The influence of the surface orientation on the force-displacement curves increases in the elastic regime until the critical stress is reached and the load drop appears. At this critical indentation depth, the orientation dependence is maximum, i.e., the forces exerted on the three principal surfaces for identical indentation vary most. Due to the stronger anisotropy of $\mathrm{Cu}$, the orientation dependence is stronger for this material than for Al. Note, however, that also in $\mathrm{Al}$, which is almost isotropic, a distinct orientation dependence is seen around the critical indentation depth; this effect is not due to the elastic anisotropy but rather to the processes of dislocation nucleation, see the discussion above. After the load drop, in both materials the force-displacement curves show almost no orientation dependence, apart form fluctuations. and increase linearly with depth.

This feature is better discussed with the help of the contact pressure, that is the force divided by the projected contact area. Note that the measurement of this area in an atomistic simulation is not without ambiguities, see the Appendix. The pertinent data are displayed in Fig. 4. Initially, before the load drop, the pressure curves increase in agreement with the $\sqrt{d}$-dependence of the Hertzian theory, Eq. (3). The pressure for the (111) and the (110) surface increase quite similarly, in agreement with the quite similar values of the indentation moduli for these two orientations, cf. Table II. The pressure on the (100) surface, however, starts deviating quite early from that of the other surfaces, and is considerably lower; this is in agreement with the above discussion of the earlier dislocation nucleation occurring under this surface. The load drop shows up in a more pronounced way in this plot, since the projected areas increase considerably during and after the nucleation of plasticity.

After the end of the load drop, the contact pressure reaches a rather constant value; this defines the hardness of the material. Thus Fig. 4 demonstrates that the surface orientation loses its influence on the hardness as soon as the plastic regime has been entered. The hardness of $\mathrm{Al}$ is measured in the simulation to be around $6 \mathrm{GPa}$, and that of $\mathrm{Cu}$ 
around $12 \mathrm{GPa}$; these values correspond quite well to the simulational data obtained in Ref. 12 . Experimental data as obtained by nanoindentation in single-crystalline $\mathrm{Cu}(100)$ give only slightly smaller values, around $6 \mathrm{GPa},{ }^{26}$ while measurements on (ultra-fine grained) poly-crystalline specimens obtain smaller hardness values, around 2 GPa. ${ }^{27}$

\section{CONCLUSIONS}

We performed simulations of nanoindentation into an elastically isotropic metal, Al, and an elastically anisotropic metal, $\mathrm{Cu}$. The three principal surfaces - the (100), (110) and (111) surface - were studied. We found:

1. The elastic deformation is fairly well described by linear elasticity theory, as tabulated by Vlassak and Nix. With increasing indentation, the effect of pressure hardening is visible.

2. Around the critical indentation depth, just before the onset of dislocation nucleation, the indentation forces show the strongest dependence on surface orientation. This dependence is almost equally strong in the elastic isotropic $\mathrm{Al}$ as in $\mathrm{Cu}$. It is not an elastic effect but due to the fact that dislocations nucleate at different global stresses due to the different orientation of the slip planes.

3. After the load drop, the contact pressure inside the material stays roughly constant and assumes the same value independently of the surface orientation. This demonstrates that the indentation hardness is a well defined concept even when the critical indentation depth has been only slightly exceeded.

\section{Acknowledgments}

The authors acknowledge financial support by the Deutsche Forschungsgemeinschaft via the Graduiertenkolleg 814 , and a generous grant of computation time from the ITWM, Kaiserslautern.

\section{APPENDIX A: HOW TO DETERMINE THE CONTACT AREA?}

The choice of the contact area is crucial for determining the contact pressure, but unfortunately not unique. On the macroscopic length scale at least two conceptionally different concepts exist: the Meyer and the Brinell contact areas, ${ }^{3}$ which represent the convex contact surface and the projection onto the initial surface plane, respectively.

For the purposes of nanoindentation, and in agreement with Hertz, the contact pressure is the substrate response in normal direction and therefore we have to choose the projected area $A^{\text {atom }}$. On the atomistic level the obvious choice to measure this area is to sum up the (projected) areas of all atoms $i$ which are in contact with the indenter, cf. Fig. 5:

$$
A^{\text {atom }}=\pi \sigma^{2} \sum_{i \in \text { contact }} \cos \alpha_{i} .
$$

Here, $\sigma$ is an 'atom radius', and $\alpha_{i}$ is the angle between the indentation direction and the vector joining the centre of the indenting sphere with atom $i$. For $\mathrm{Cu}$ we choose $\sigma=1.35 \AA$. Unfortunately, this definition leads to serious problems, since $A^{\text {atom }}$ describes a non-connected area; indeed, between the atoms there appear 'holes'. This becomes an important problem in particular in the elastic regime, where due to the elastic deformation, these 'holes' increase in size with increasing deformation, leading to systematic errors: the area is underestimated, and the pressure is overestimated. A further systematic effect shows up due to the different areal densities of surface planes: For atomically rough surfaces, such as the (110), the area is underestimated in comparison to dense surfaces, such as the (111); this effect leads to a distortion of hardness determinations from simulation data for different surfaces. Fig. 6 demonstrates these effects: besides a general overestimation of the contact area, the order of the stiffness for the (111) and the (110) surfaces is exchanged.

In the present paper, we therefore have chosen an elliptic contact area, which is connected and conserves the right order of the pressures:

$$
A^{\text {elliptic }}=\frac{\pi}{4}\left(x^{\max }-x^{\min }\right)\left(y^{\max }-y^{\min }\right) .
$$


TABLE I: Elastic properties of $\mathrm{Al}$ and $\mathrm{Cu}$ as described by the potentials by Zhou et al. ${ }^{16}$ and by Mishin et al. ${ }^{17}$. Elastic constants $c_{i j}$, elastic anisotropy $X$, Eq. (5), bulk modulus $B$, average shear modulus $G$, and average Poisson ratio $\nu$.

\begin{tabular}{l|lll|l|lll} 
& $c_{11}(\mathrm{GPa})$ & $c_{12}(\mathrm{GPa})$ & $c_{44}(\mathrm{GPa})$ & $X$ & $B(\mathrm{GPa})$ & $G(\mathrm{GPa})$ & $\nu$ \\
\hline $\mathrm{Al}$ & 114 & 61.5 & 31.6 & 1.20 & 79.0 & 29.3 & 0.33 \\
$\mathrm{Cu}$ & 169.9 & 122.6 & 76.2 & 3.22 & 138.4 & 47.8 & 0.35
\end{tabular}

Here, $x$ and $y$ measure the coordinates of the contact atoms projected into the initial surface plane. This set of coordinates describes a curved contour line, which is approximated by an ellipse. In Eq. (A2), $x^{\max }-x^{\min }$ and $y^{\max }-y^{\min }$ denote the major and minor diameters of this ellipse, respectively. This definition leads initially, i.e., when the indenter has contact with few atoms only, to an increased noise in the determination of the area and hence the contact pressure, cf. Fig. 4. For larger indentations, however, it is more reliable.

* Electronic address: urbassek@rhrk.uni-kl.de; URL: http://www.physik.uni-kl.de/urbassek/

1 H. Hertz, J. reine und angewandte Mathematik 92, 156 (1882).

2 L. D. Landau and E. M. Lifshitz, Theory of elastictiy, vol. 7 of Course of Theoretical Physics (Pergamon, Oxford, 1970), 2nd ed.

3 A. C. Fischer-Cripps, Nanoindentation (Springer, New York, 2004), 2nd ed.

4 A. C. Fischer-Cripps, Introduction to Contact Mechanics (Springer, New York, 2007), 2nd ed.

5 J. D. Kiely and J. E. Houston, Phys. Rev. B 57, 12588 (1998).

6 J. D. Kiely, K. F. Jarausch, J. E. Houston, and P. E. Russell, J. Mater. Res. 14, 2219 (1999).

7 J. R. Willis, J. Mech. Phys. Sol. 14, 163 (1966).

8 J. R. Willis, J. Mech. Phys. Sol. 15, 331 (1967).

9 J. J. Vlassak and W. D. Nix, Philos. Mag. A 67, 1045 (1993).

10 J. J. Vlassak and W. D. Nix, J. Mech. Phys. Sol. 42, 1223 (1994).

11 T. Tsuru and Y. Shibutani, Modelling Simul. Mater. Sci. Eng. 14, S55 (2006).

12 T. Tsuru and Y. Shibutani, Phys. Rev. B 75, 035415 (2007).

13 K. J. Van Vliet, J. Li, T. Zhu, S. Yip, and S. Suresh, Phys. Rev. B 67, 104105 (2003).

14 M. A. Tschopp, D. E. Spearot, and D. L. McDowell, Modelling Simul. Mater. Sci. Eng. 15, 693 (2007).

15 X. H. Liu, J. F. Gu, Y. Shen, and C. F. Chen, Scr. Materialia 58, 564 (2008).

${ }^{16}$ X. W. Zhou, H. N. G. Wadley, R. A. Johnson, D. J. Larson, N. Tabat, A. Cerezo, A. K. Petford-Long, G. D. W. Smith, P. H. Clifton, R. L. Martens, et al., Acta Mater. 49, 4005 (2001).

17 Y. Mishin, M. J. Mehl, D. A. Papaconstantopoulos, A. F. Voter, and J. D. Kress, Phys. Rev. B 63, 224106 (2001).

18 C. L. Kelchner, S. J. Plimpton, and J. C. Hamilton, Phys. Rev. B 58, 11085 (1998).

19 http://lammps.sandia.gov/.

20 D. Christopher, R. Smith, and A. Richter, Nucl. Instrum. Meth. B 180, 117 (2001).

21 X.-L. Ma and W. Yang, Nanotechnology 14, 1208 (2003).

22 J. Turley and G. Sines, J. Phys. D 4, 264 (1971).

${ }^{23}$ T. Zhu, J. Li, K. J. Van Vliet, S. Ogata, S. Yip, and S. Suresh, J. Mech. Phys. Sol. 52, 691 (2004).

24 S. Ogata, J. Li, and S. Yip, Science 298, 807 (2002).

25 M. A. Tschopp and D. L. McDowell, J. Mech. Phys. Sol. 56, 1806 (2008).

26 M. Göken and M. Kempf, Z. f. Metallkunde 92, 1061 (2001).

27 K. Durst, B. Backes, and M. Göken, Scr. Mater. 52, 1093 (2005). 
TABLE II: Orientation dependent indentation moduli. $M^{\text {theo }}$ : after Vlassak and $\mathrm{Nix}^{9,10}$.

$M^{\text {appr }}$ : obtained according to the simplified recipe, Eq. (12).

$M^{\mathrm{MD} \text {,init }}$ : from a fit to our MD results over $d<2 \AA$.

$M^{\mathrm{MD} \text {,full }}$ : from a fit to our MD results over the full elastic region.

The isotropic indentation modulus $M^{\text {iso }}$ as calculated from the isotropic constants, Eqs. (9) - (11), appropriate for a polycrystal, is given as a reference.

\begin{tabular}{l|llll|lll|}
\multirow{2}{*}{} & \multicolumn{3}{|c|}{$\mathrm{Al}$} & \multicolumn{3}{c|}{$\mathrm{Cu}$} \\
\cline { 2 - 7 } & $(100)$ & $(110)$ & $(111)$ & $(100)$ & $(110)$ & $(111)$ \\
\hline$M^{\text {theo }}(\mathrm{GPa})$ & 87.0 & 88.4 & 88.9 & 135.0 & 148.0 & 151.9 \\
$M^{\text {appr }}(\mathrm{GPa})$ & 80.5 & 90.3 & 93.4 & 81.4 & 179 & 211 \\
\hline$M^{\mathrm{MD}, \text { init }}(\mathrm{GPa})$ & 92 & 96 & 101 & 134 & 155 & 171 \\
$M^{\mathrm{MD}, \text { full }}(\mathrm{GPa})$ & 95 & 97 & 106 & 147 & 176 & 192 \\
\hline$M^{1 \text { 1So }}(\mathrm{GPa})$ & \multicolumn{3}{|c|}{88.2} & & \multicolumn{3}{c|}{145.9}
\end{tabular}
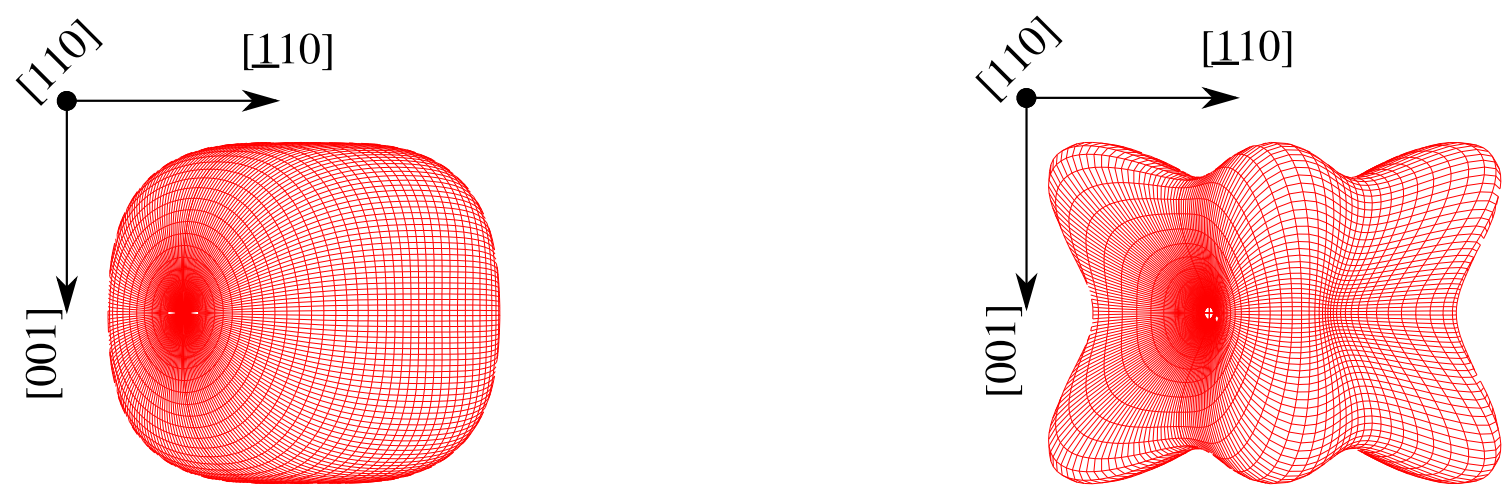

(a)

(b)

FIG. 1: Polar plot of the orientation dependence of Young's modulus, for $\mathrm{Al}$ (a) and $\mathrm{Cu}$ (b), Eq. (6). The view direction is aligned with the [110] direction.

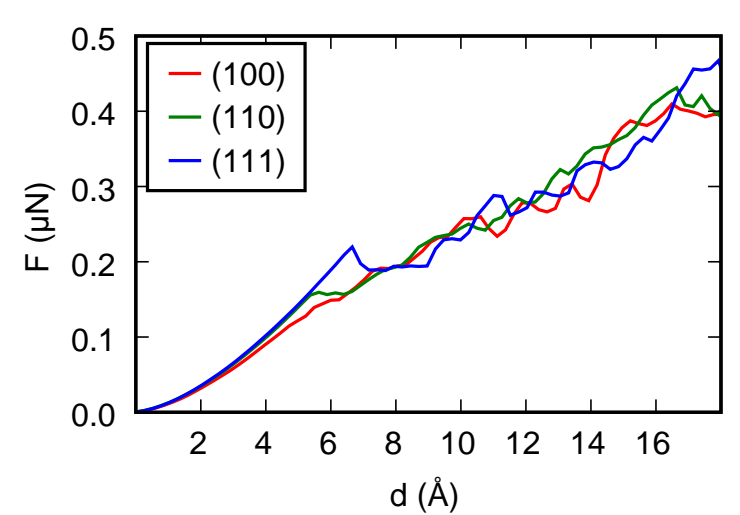

(a)

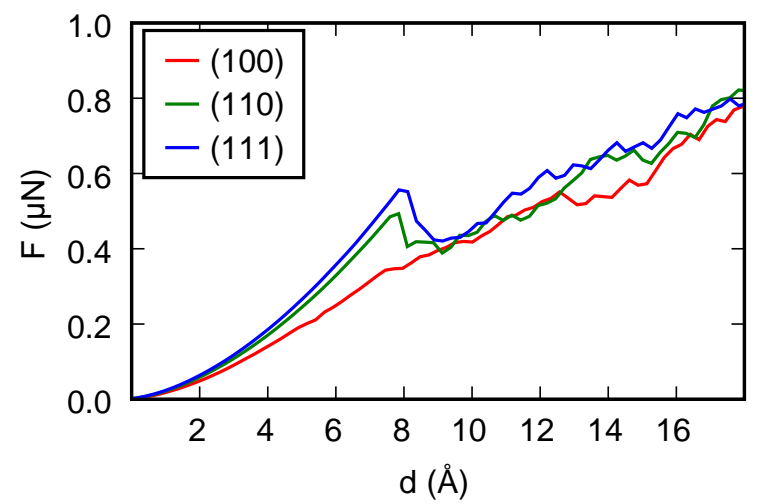

(b)

FIG. 2: Force $F$ vs displacement $d$ in $\mathrm{Al}$ (a) and $\mathrm{Cu}$ (b) for the three surface orientations studied. 


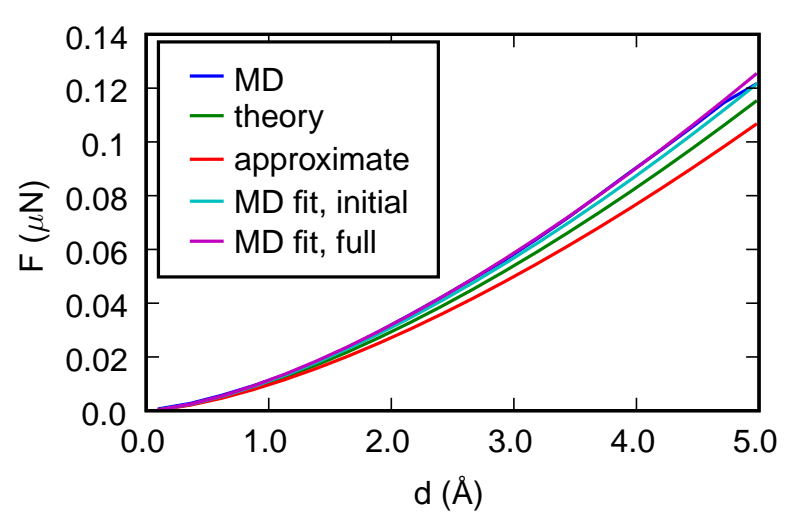

(a)

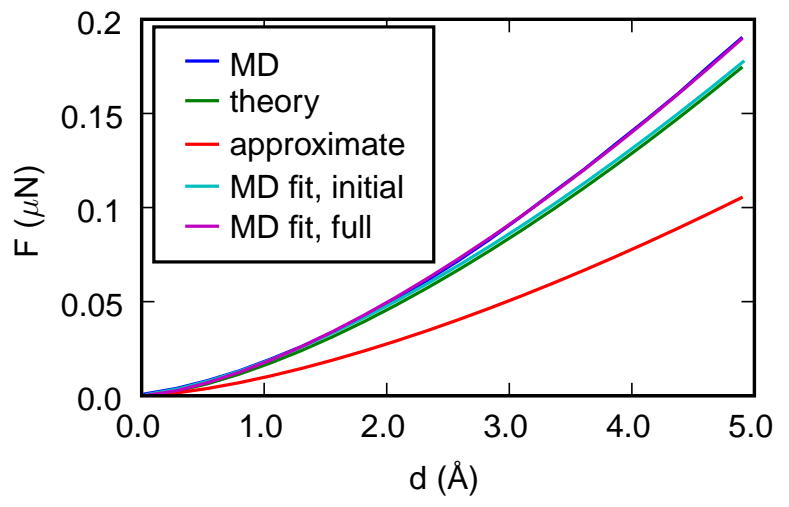

(b)

FIG. 3: Comparison of the force $F$ vs displacement $d$ data as obtained by molecular dynamics (MD) with various theories and fits. theory: theoretical prediction for anisotropic media by Vlassak and Nix. ${ }^{9,10}$ approximate: Eq. (12).

MD fit, initial: Fit of MD data to Hertz' law, Eq. (1), for $d<2 \AA$.

MD fit, full: Fit of MD data to Hertz' law, Eq. (1), for the full elastic region.

a) $\mathrm{Al}(100)$. b) $\mathrm{Cu}(100)$.

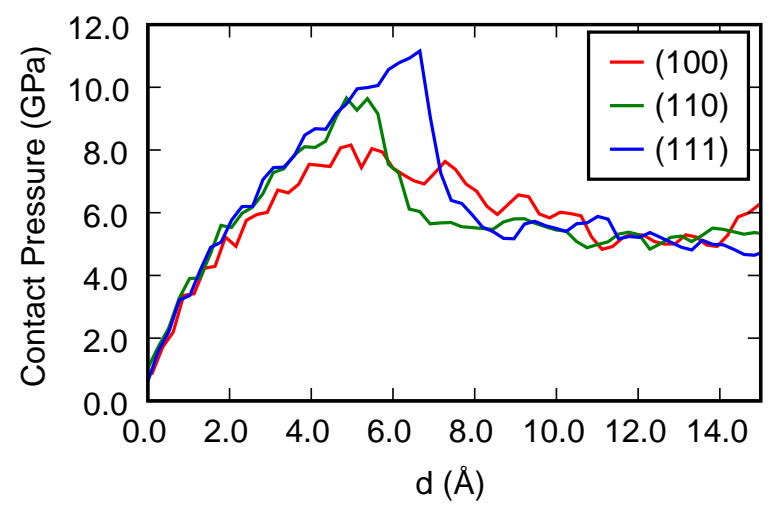

(a)

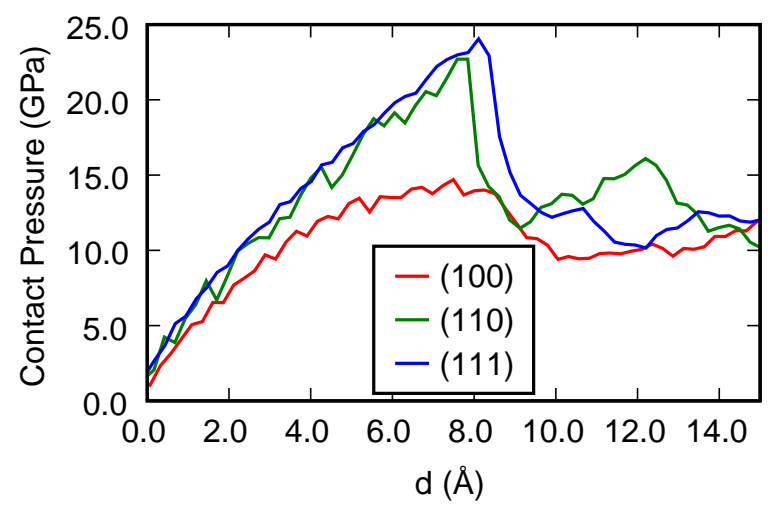

(b)

FIG. 4: Contact pressure vs displacement $d$ in $\mathrm{Al}(\mathrm{a})$ and $\mathrm{Cu}(\mathrm{b})$ for the three surface orientations studied.

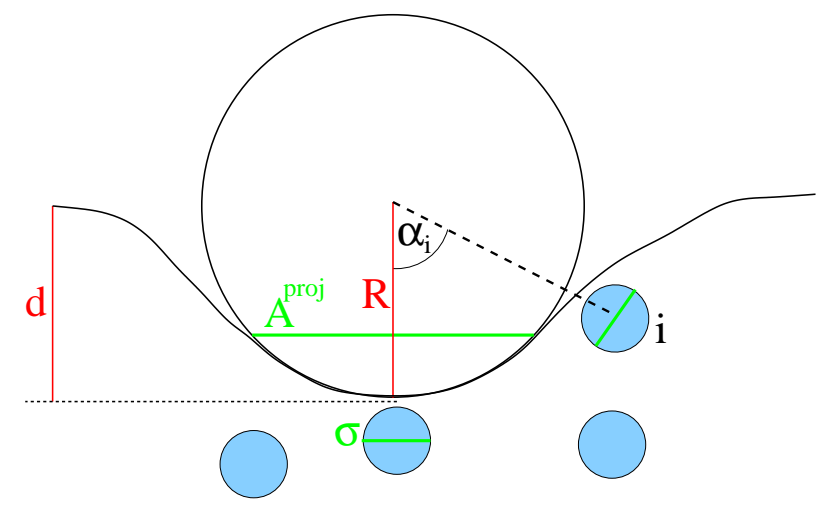

FIG. 5: Schematics of nanoindentation by a sphere of radius $R$. The indentation depth $d$ is measured with respect to the initial surface plane. The relevant area $A^{\text {proj }}$ is the contact area of the indenter with the substrate projected into the initial surface plane. $\alpha_{i}$ is the angle between the indentation direction and the vector joining the centre of the indenting sphere with atom $i$. 


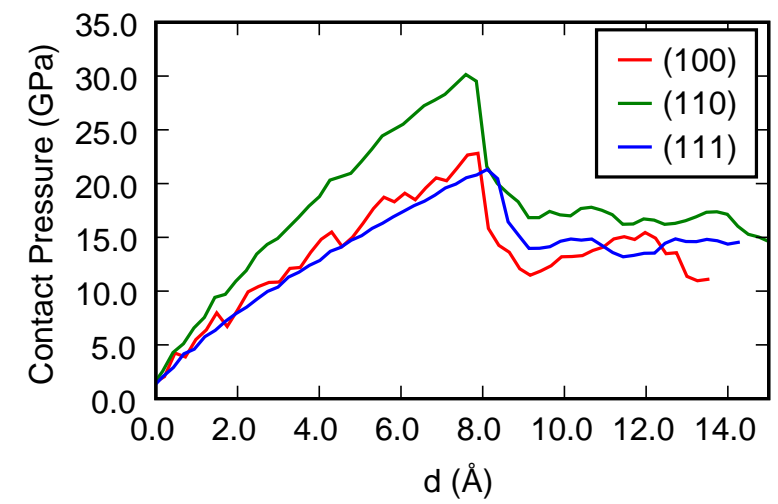

FIG. 6: Contact pressure vs displacement $d$ in $\mathrm{Cu}$ for the three surface orientations studied, as measured with a too simplistic definition of the indenter contact area, Eq. (A1). 


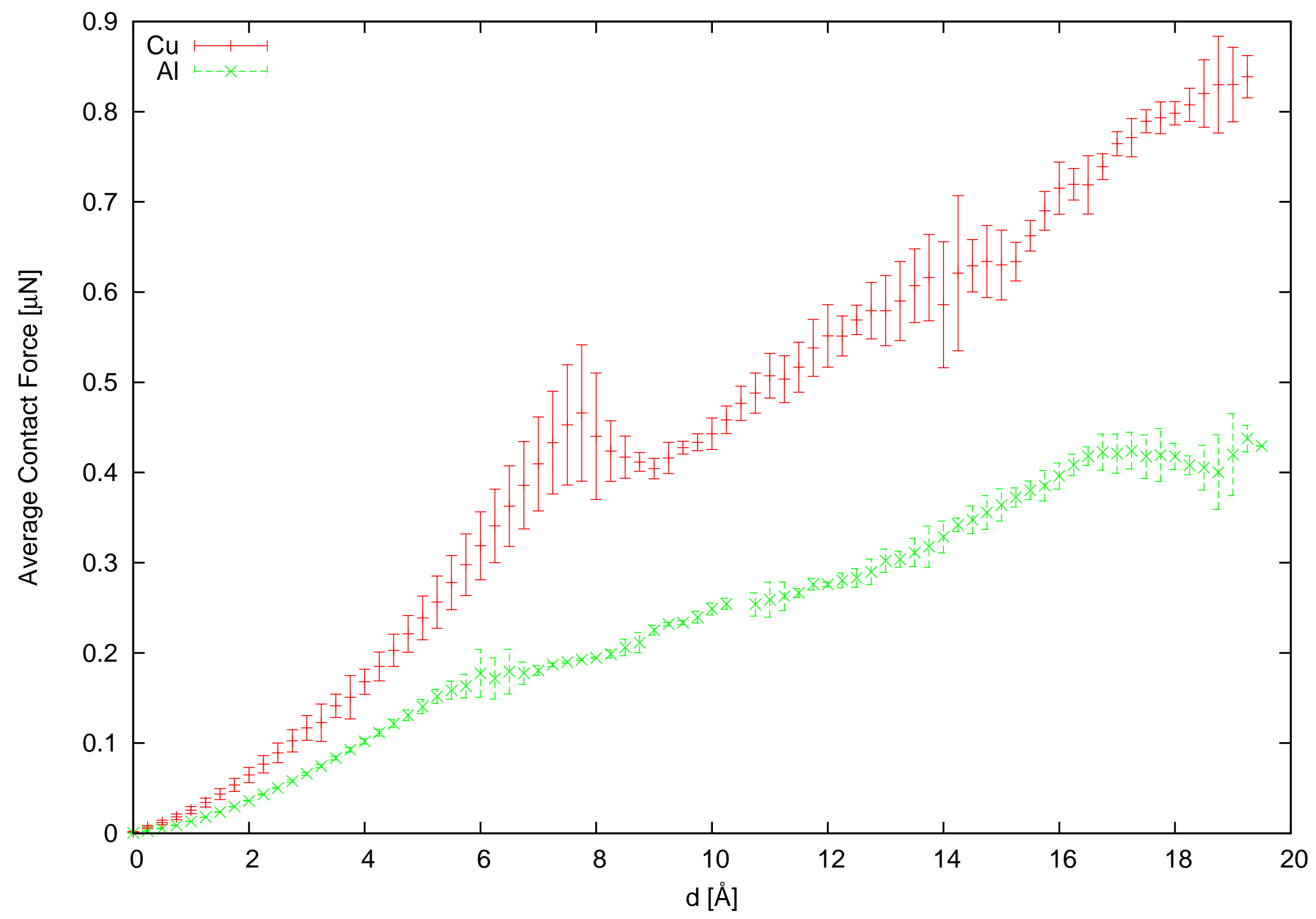




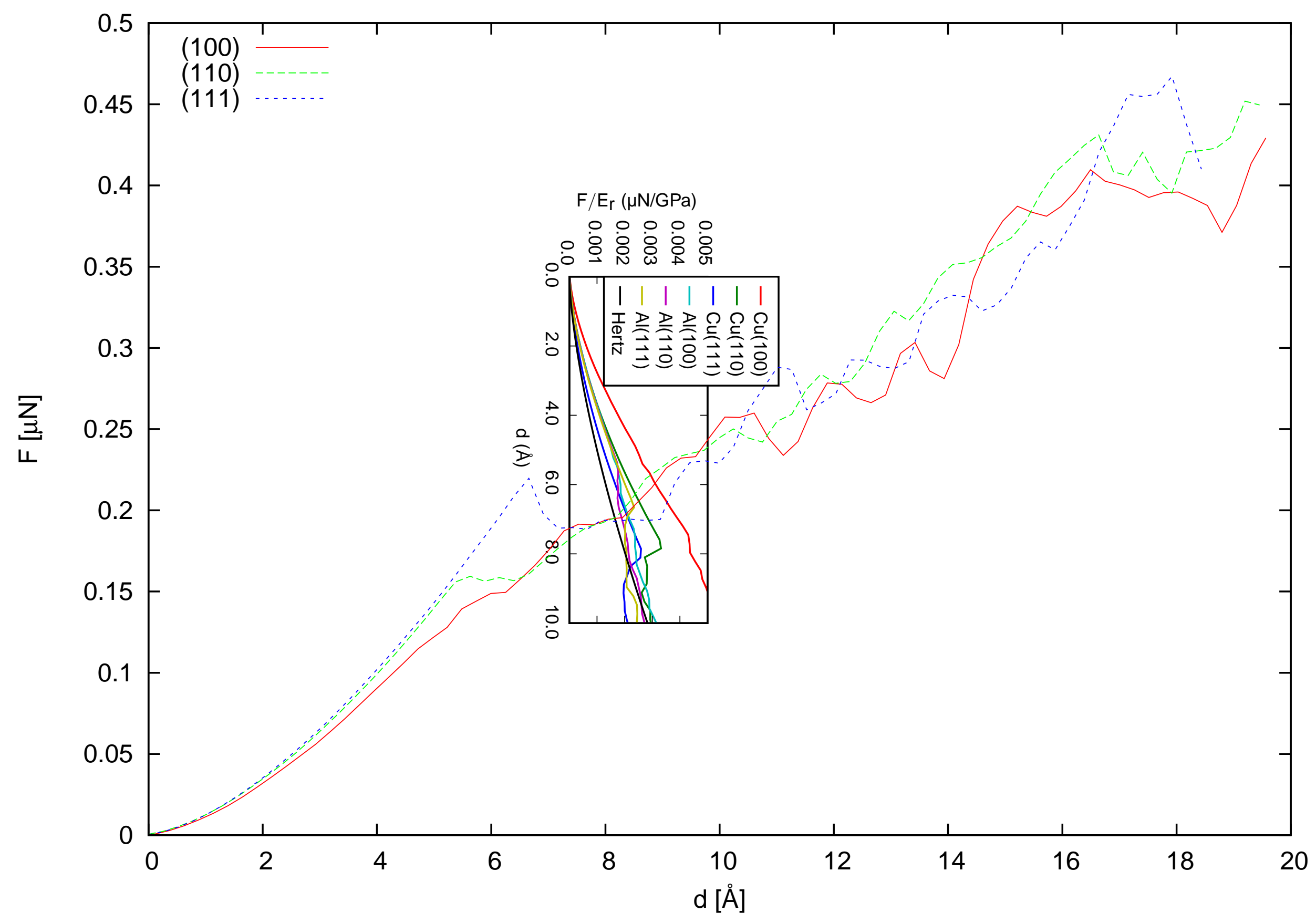




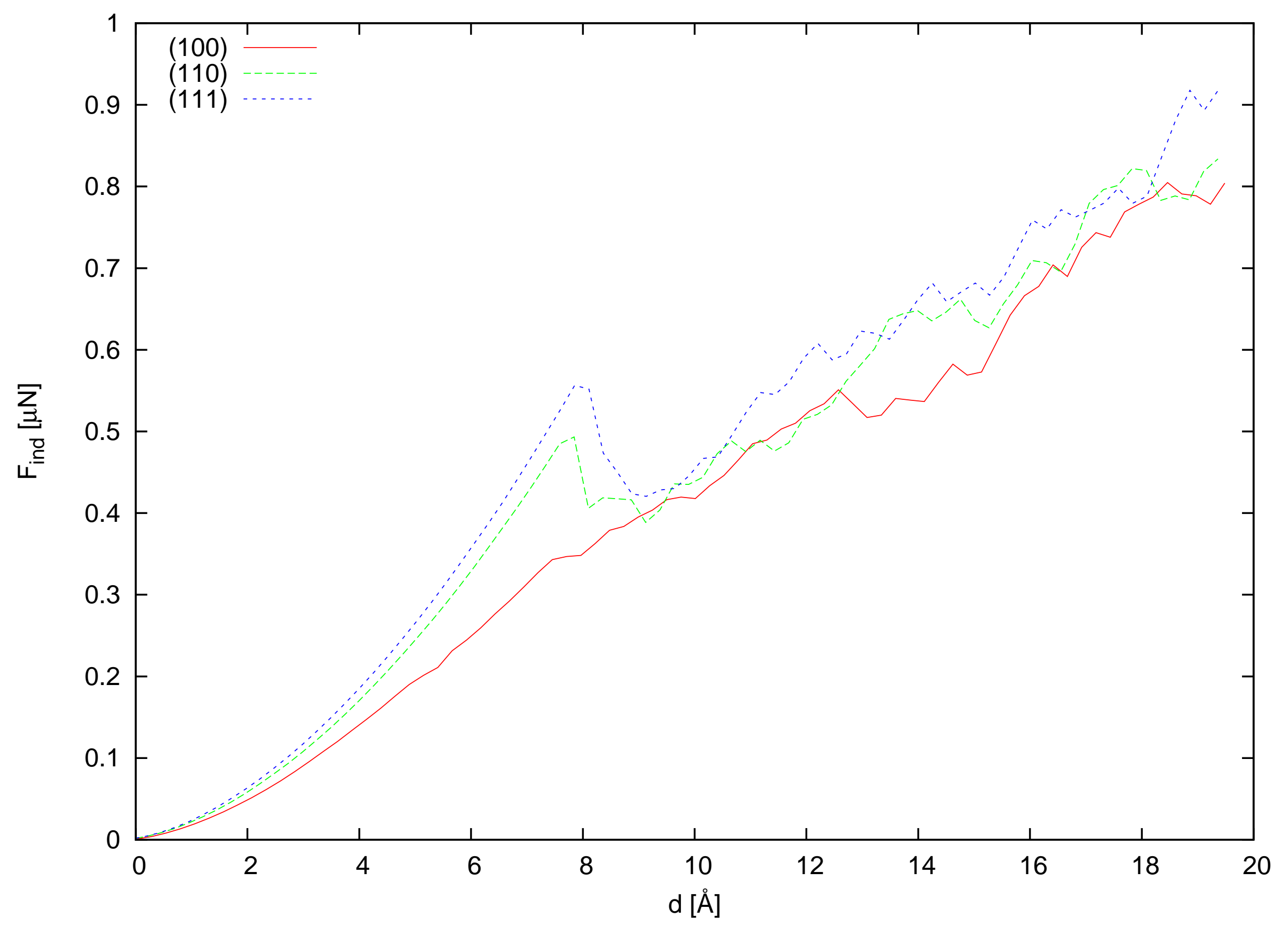




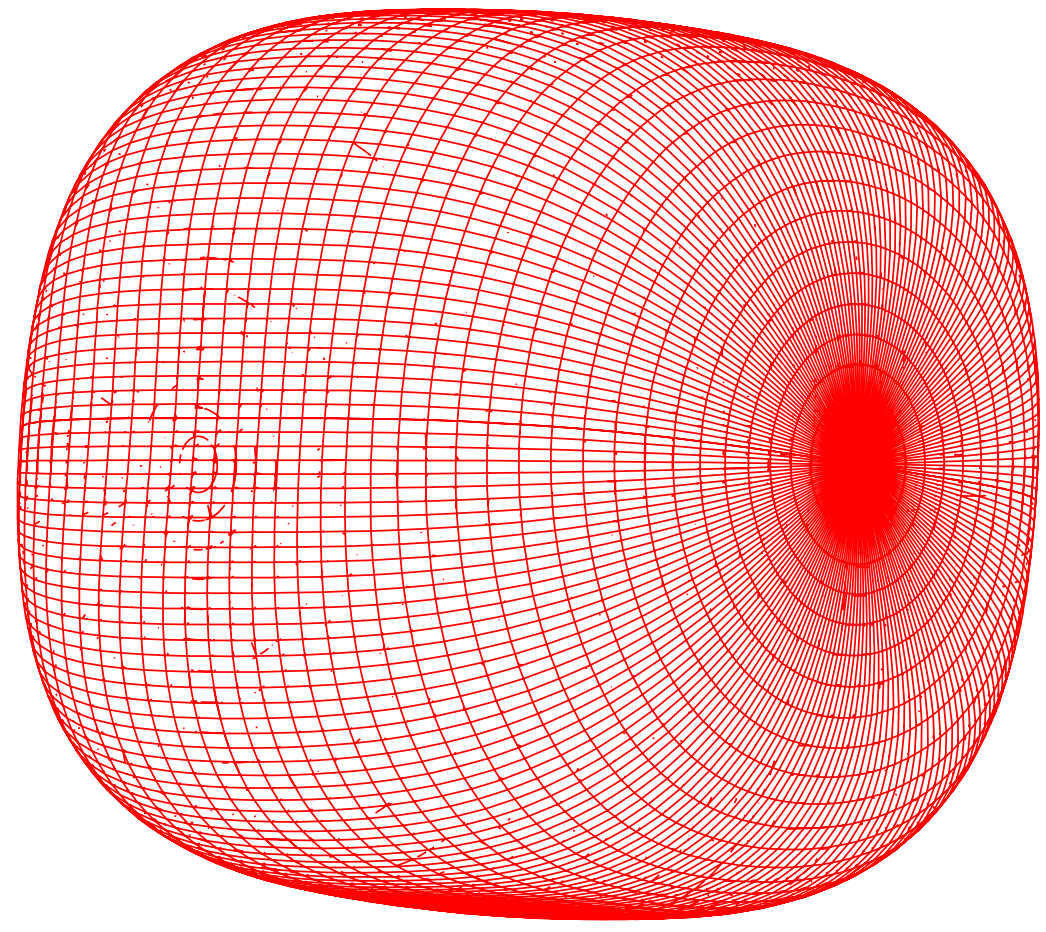




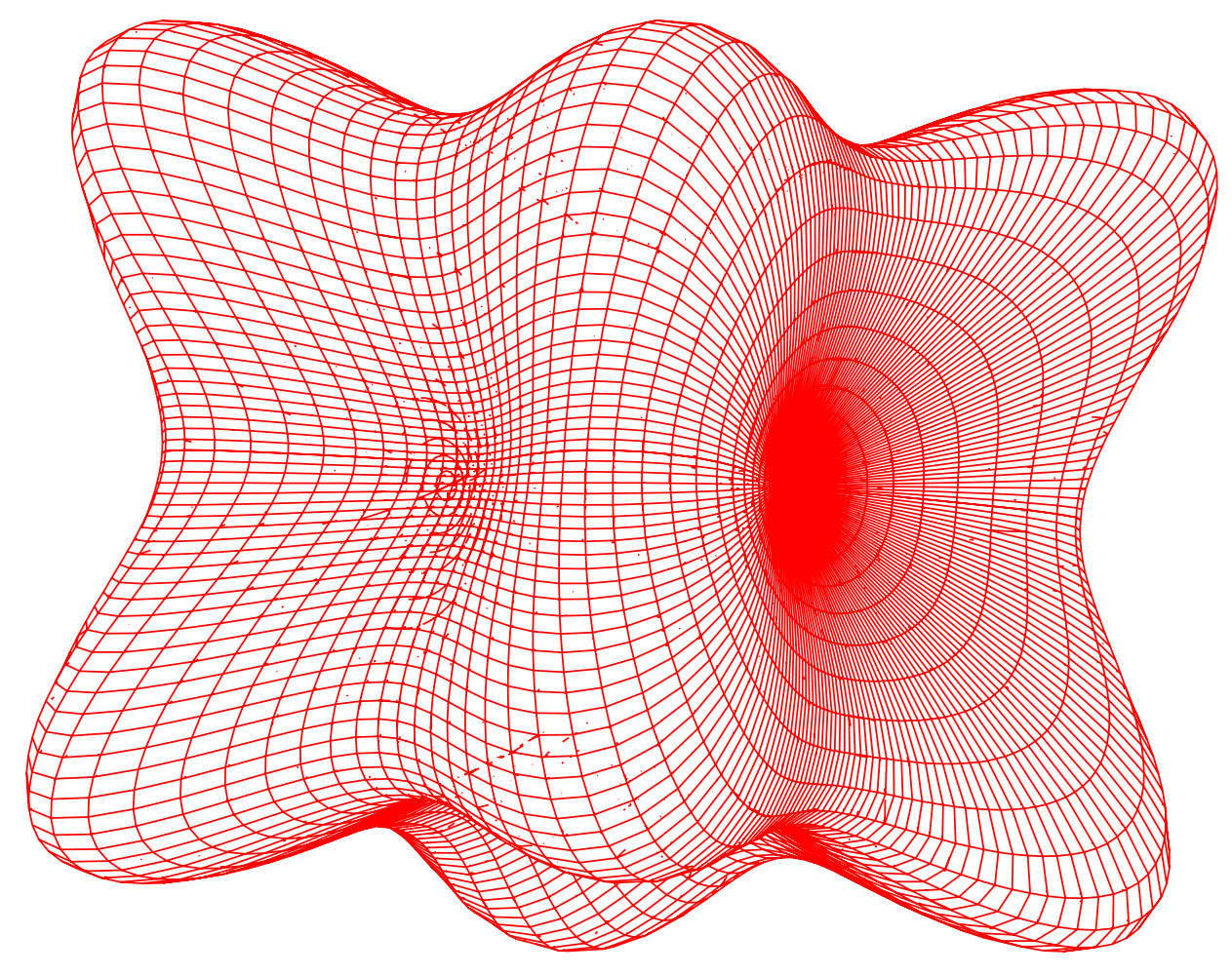




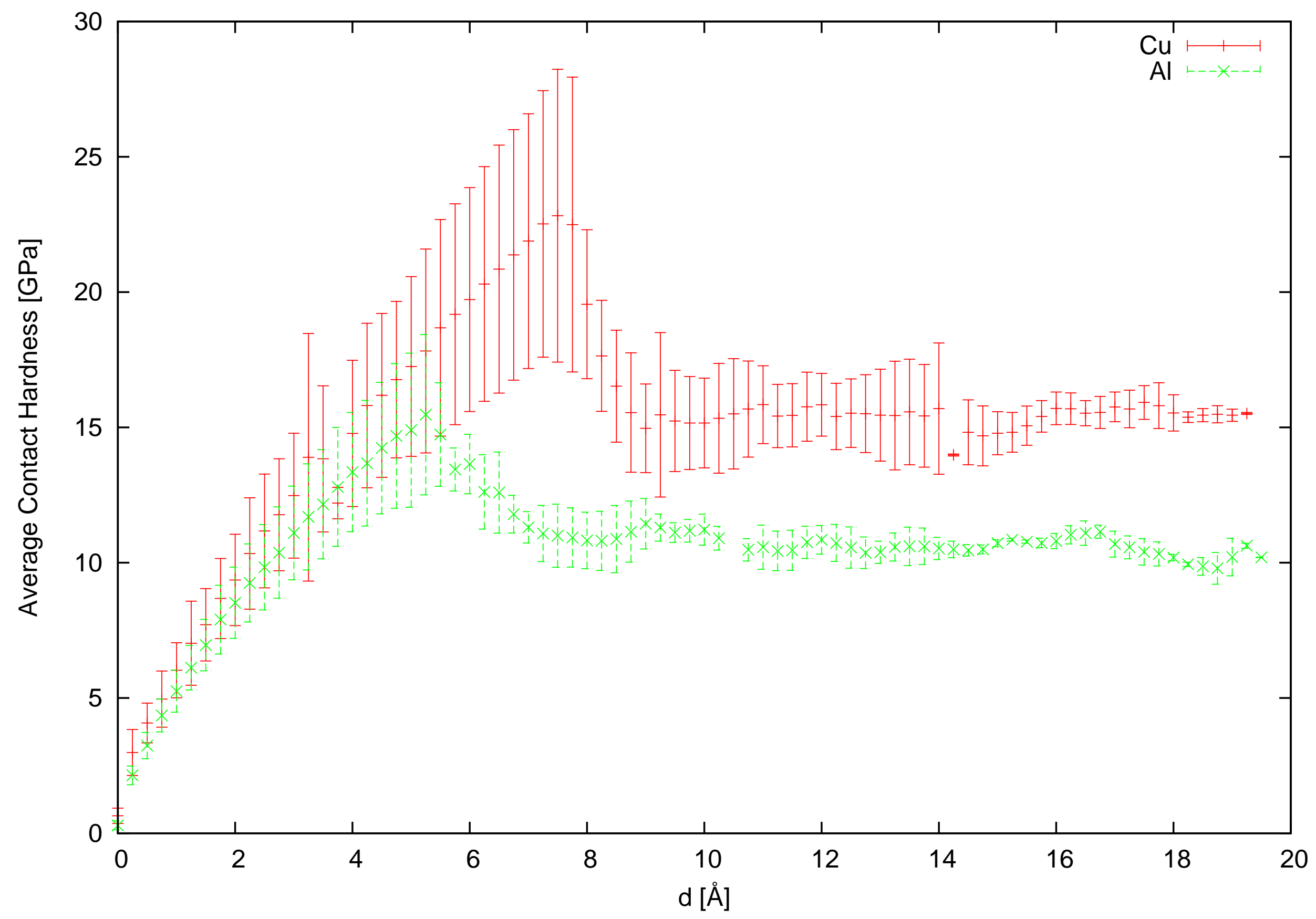




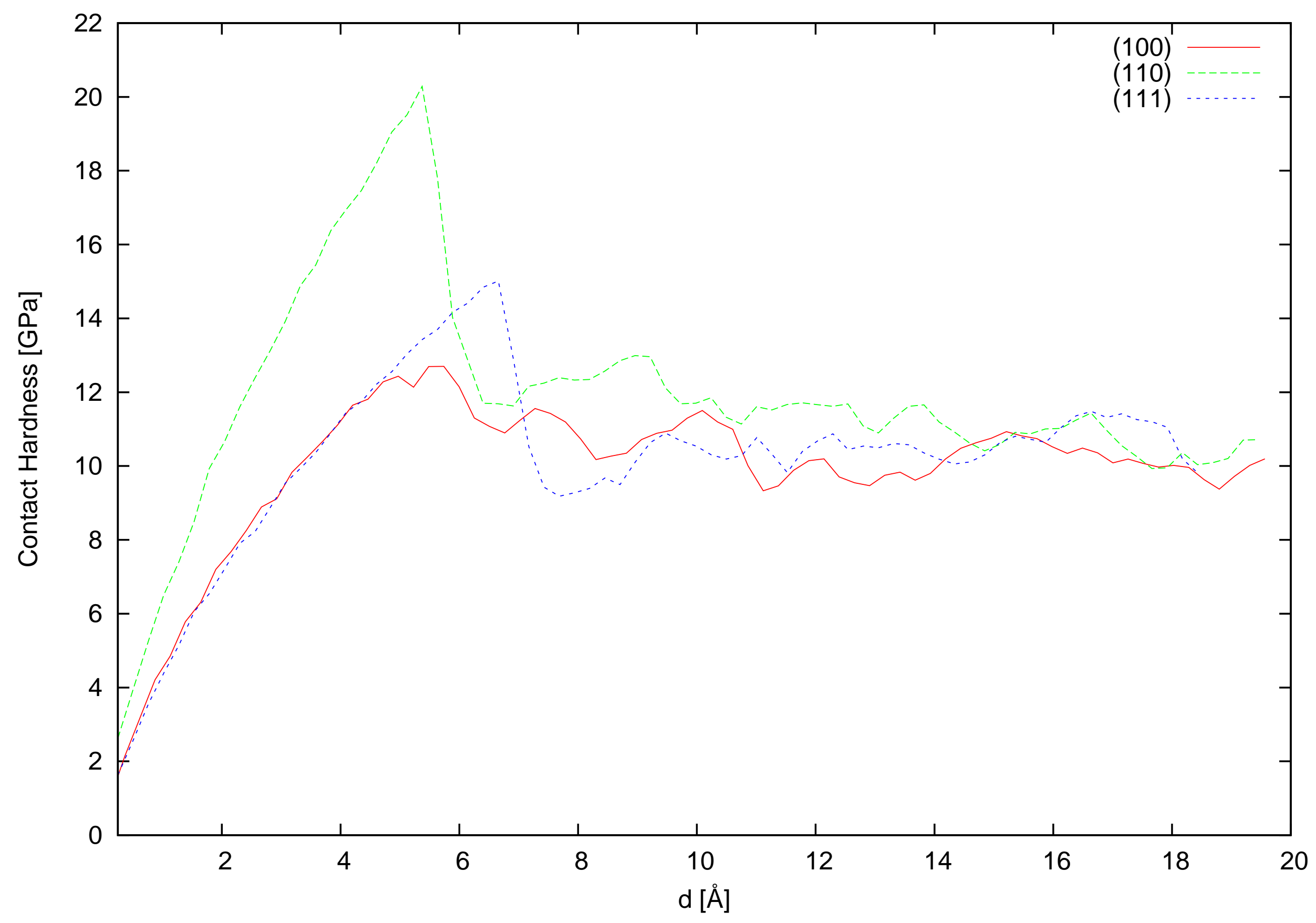




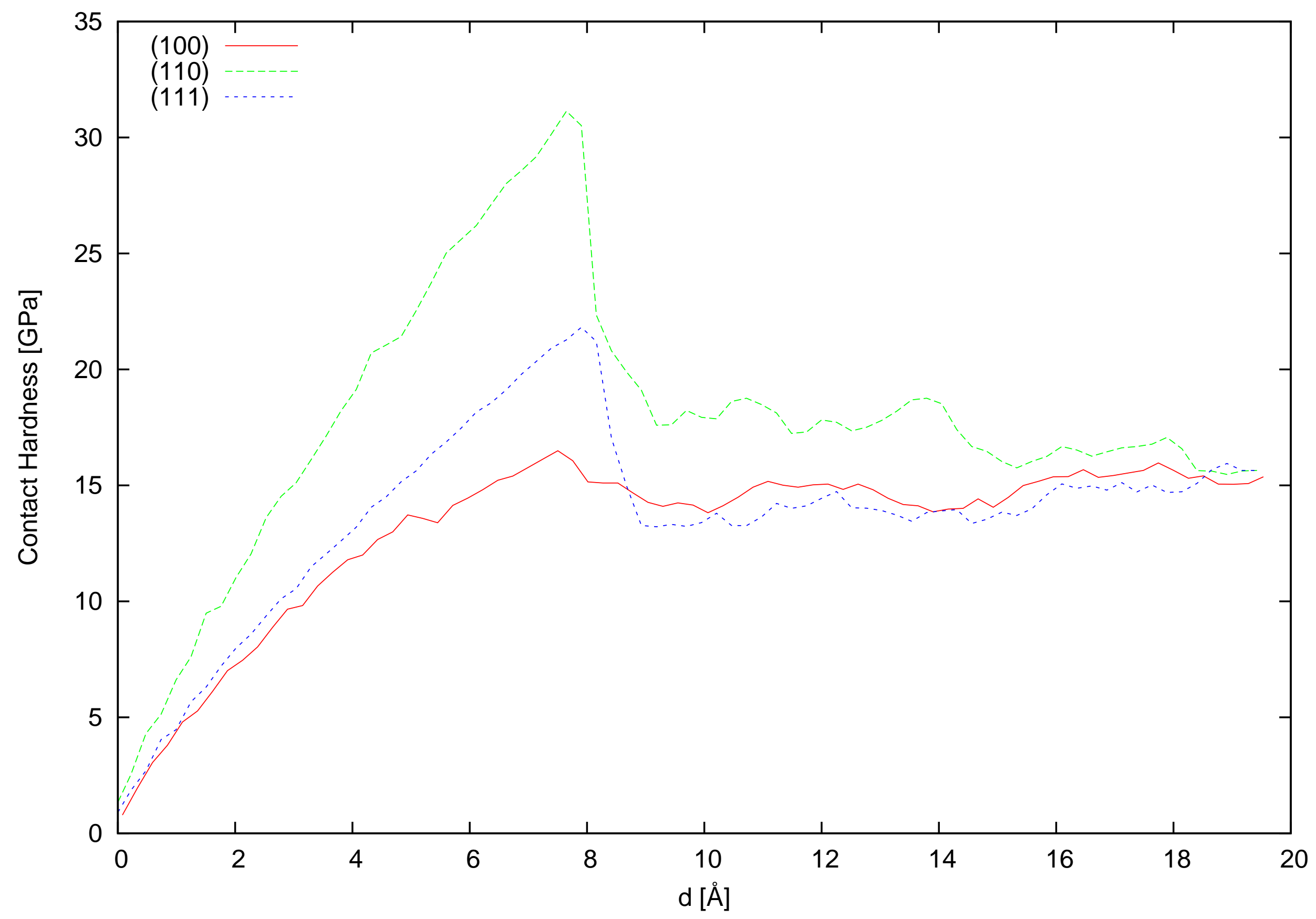




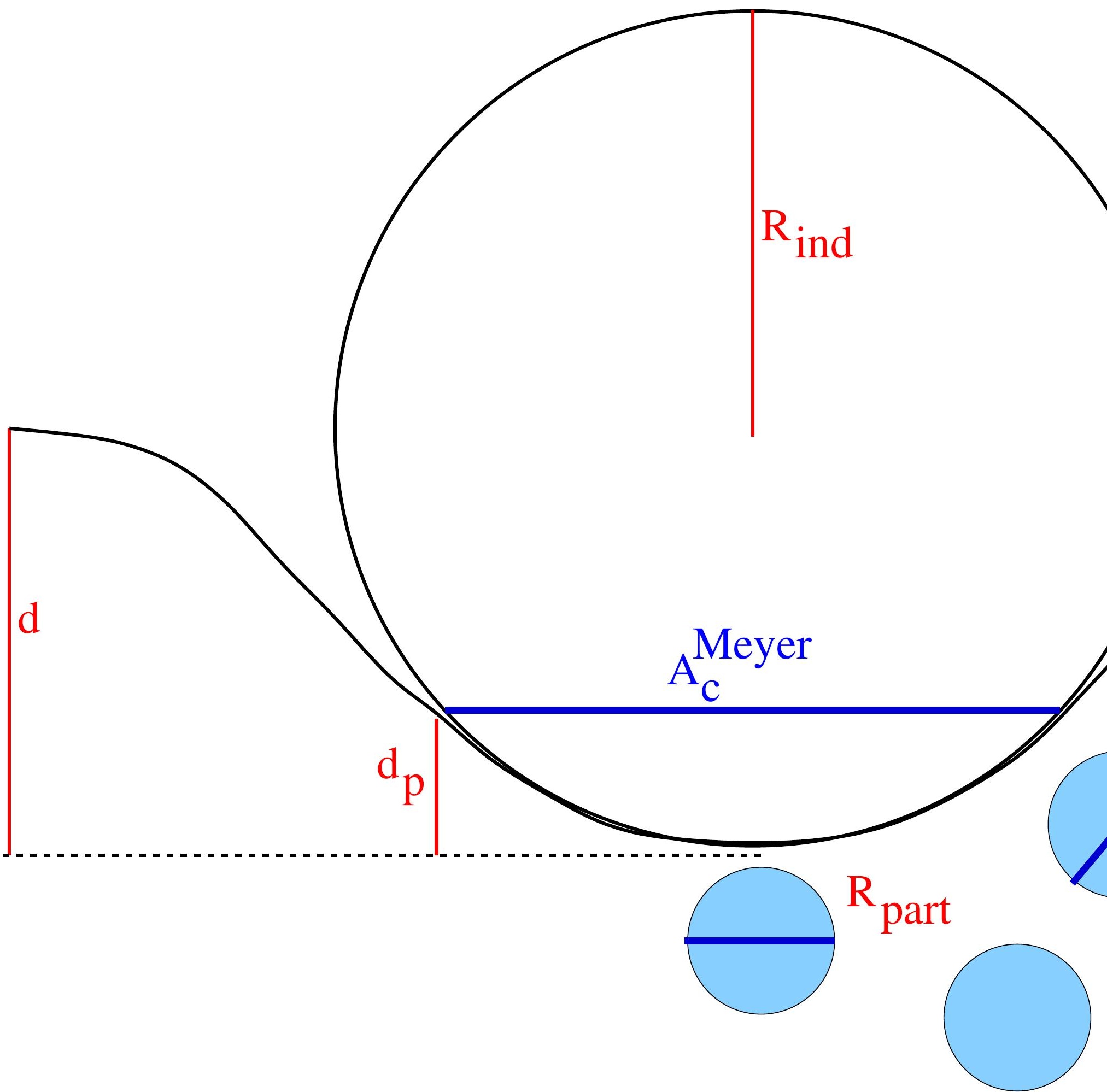

